\title{
Combined Metabolically Active Tumor Volume and Early Metabolic Response Improve Outcome Prediction in Metastatic Colorectal Cancer
}

Erwin Woff $^{1}$, Lisa Salvatore ${ }^{2}$, Federica Marmorino ${ }^{3}$, Dario Genovesi ${ }^{4}$, Gabriela Critchi ${ }^{1}$, Thomas Guiot $^{1}$, Lieveke Ameye $^{5}$, Francesco Sclafani $^{6}$, Alain Hendlisz $^{6 *}$, Patrick Flamen $^{1 *}$

* Patrick Flamen and Alain Hendlisz contributed equally to this work.

1 Nuclear Medicine Department, Institut Jules Bordet - Université Libre de Bruxelles (ULB), Brussels, Belgium.

2 Medical Oncology Department, Comprehensive Cancer Center, Fondazione Policlinico Universitario Agostino Gemelli IRCCS, Rome, Italy.

3 Department of Translational Research and New Technologies in Medicine and Surgery, Unit of Medical Oncology 2, Azienda Ospedaliera Universitaria Pisana, Pisa, Italy.

4 Nuclear Medicine Department, Fondazione Toscana "Gabriele Monasterio", Pisa, Italy.

5 Data centre, Institut Jules Bordet - Université Libre de Bruxelles (ULB), Brussels, Belgium. 6 Medical Oncology Department, Institut Jules Bordet - Université Libre de Bruxelles (ULB), Brussels, Belgium. 


\section{Corresponding and first author:}

Dr Erwin Woff, MD, PhD

Nuclear Medicine Department,

Institut Jules Bordet - Université Libre de Bruxelles (ULB),

1 rue Héger-Bordet, 1000 Brussels, Belgium.

Tel: +322/5417314; Fax: +322/5413224; E-mail: erwin.woff@ bordet.be

Content and word count (Microsoft Word 2016 MSO): Abstract: 301/350; Word count of:

title page, abstract, text, disclosure, acknowledgments, key points, references, figure legends, and tables: 5092; Figures: 5; Tables: 2; Supplemental Figure: 1, Supplemental Tables: 3, References: 20

\section{Running title:}

WB-MATV \& metabolic response in mCRC 


\section{ABSTRACT}

Stratification of metastatic colorectal cancer (mCRC) patients is mostly based on clinical and biological characteristics. This study aimed to validate the prognostic value of ${ }^{18} \mathrm{~F}$-FDG PET/CTbased biomarkers such as baseline whole-body metabolically active tumor volume (WB-MATV) and early metabolic response (mR) in mCRC.

\section{Methods}

The development cohort included chemorefractory mCRC patients enrolled in two prospective Belgian multicenter trials evaluating last-line treatments (multikinase inhibitors). The validation cohort included mCRC patients from an Italian center treated with chemotherapy and bevacizumab as first-line. Baseline WB-MATV was defined as the sum of metabolically active volumes of all target lesions identified on the baseline ${ }^{18} \mathrm{~F}$-FDG PET/CT. Early metabolic response $(\mathrm{mR})$ assessment was performed following usual response criteria (PERCIST-30\%, PERCIST-15\%, EORTC) and the so-called CONSIST method, which defines response as a decrease of SULmax $\geq 15 \%$ for all target lesions. Baseline WB-MATV and early mR assessment were investigated along with usual clinical factors and correlated with overall and progressionfree survival (OS/PFS).

\section{Results}

Clinical factors, baseline WB-MATV and early mR were evaluable in 192/239 and 94/125 patients of the development and validation cohorts, respectively. Except for PERCIST-30\%, all response methods were equivalent in terms of outcome prediction and CONSIST was found to be the most accurate. Baseline WB-MATV and early mR using CONSIST method were independent prognostic parameters after adjustment for clinical factors in the development and validation sets 
for both OS (HR WB-MATV: 1.87 (1.17-2.97), $P=0.005$, and HR early mR: 1.79 (1.08-2.95), $P$ $=0.02$ for the validation set), and PFS (HR WB-MATV: $1.94(1.27-2.97), P=0.002$, and HR early mR: $1.69(1.04-2.73), P=0.03$ for the validation set).

\section{Conclusion}

Baseline WB-MATV and early $\mathrm{mR}$ are strong independent prognostic biomarkers for OS/PFS in mCRC, regardless of treatment received. Therefore, combining these biomarkers improves risk stratification for OS/PFS in mCRC.

Key Words: ${ }^{18}$ F-FDG PET/CT-based biomarkers, metabolically active tumor volume, early metabolic response, metastatic colorectal cancer. 


\section{INTRODUCTION}

Despite significant improvements over the last 15 years, patients with metastatic colorectal cancer (mCRC) still hold a poor prognosis with a 5-y survival rate less than $15 \%$ (1). Nevertheless, survival differs significantly among patients, creating the need for prognostic biomarkers to improve patient stratification and personalized care.

Baseline whole-body metabolically active tumor volume (WB-MATV), an ${ }^{18}$ F-FDG PETbased quantitative parameter, has recently been reported by our group to be a strong independent prognostic imaging biomarker in chemorefractory mCRC with a higher prognostic value than the usual clinical prognostic factors (2). However, these findings still required validation in mCRC patients undergoing first-line treatment.

Early metabolic response $(\mathrm{mR})$ assessment using ${ }^{18} \mathrm{~F}-\mathrm{FDG}$ PET/CT is a valuable tool for the rapid identification of patients with treatment resistant tumors, faster than with conventional, morphology-based imaging (CT/MRI). It has also been shown to be a strong predictor of outcome in many tumor types $(3,4)$. The high negative predictive value of early $\mathrm{mR}$ assessment (performed as early as after one treatment cycle) is a key strength of metabolic imaging, essential to avoid pursuing ineffective and potentially toxic treatments, thereby allowing a rapid and costeffective way to reallocate societal resources towards more promising therapies $(3,5)$. To our knowledge, no prospective validation study has been reported so far on the predictive value of early $\mathrm{mR}$ assessment and its independence from baseline WB-MATV and clinical prognostic factors in mCRC. 
Different metabolic response assessment criteria have been explored in many cancer types including mCRC, but until now, no consensus has been reached on which criteria is best to use and whether these different response criteria are equivalent in terms of outcome prediction $(6,7)$.

The aims of this study were: first, to validate the prognostic value of baseline WB-MATV and early $\mathrm{mR}$ assessment in chemonaïve mCRC patients; second, to assess whether early $\mathrm{mR}$ yields additional predictive value when combined with clinical factors and baseline WB-MATV; and last, to evaluate the relative predictive values of the usual metabolic response criteria. 


\section{MATERIALS AND METHODS}

\section{Study Population}

This study included mCRC patients from three prospective data sets. The development set was composed of two Belgian multicenter single-arm phase II trials: SoMore and RegARd-C, which have already been described in a previous report (2). These trials were conducted on chemorefractory mCRC patients $(n=239)$ treated with capecitabine/sorafenib (SoMore) or regorafenib (RegARd-C). The external validation set consisted of an Italian monocentric singlearm study. This study investigated the correlation between early $\mathrm{mR}$ and survival outcomes (overall survival $[\mathrm{OS}]$ and progression-free survival $[\mathrm{PFS}]$ ) in chemonaïve mCRC patients $(n=125)$ treated with standard first-line chemotherapy combined with targeted agents $(8)$.

Patient eligibility criteria and study design for the first two data sets were previously reported $(9,10)$ but can be described shortly as follows: histologically proven colon or rectum adenocarcinoma; tumor refractory to all standard chemotherapy agents; age greater than 18 y; Eastern Cooperative Oncology Group performance status of 1 or less; life expectancy greater than $12 \mathrm{wk}$; a baseline (before treatment start) and an early ${ }^{18} \mathrm{~F}-\mathrm{FDG}$ PET/CT (after 2-3 weeks of therapy) with at least one measurable target lesion on the baseline examination; a minimum washout period of $4 \mathrm{wk}$ before inclusion in the trial; and provision of signed informed consent. Eligibility criteria for the external validation set were the same except that all patients were chemonaïve.

Ethics approvals for these three trials were obtained from the relevant local ethical committee of each center. All procedures performed in this study involving human participants 
were in accordance with the ethical standards of the institutional or national research committee and the 1964 Helsinki declaration and its later amendments or comparable ethical standards.

\section{${ }^{18}$ F-FDG PET/CT Imaging}

Eight Belgian EARL-accredited and one Italian PET/CT centers were involved in this study, with each following strict procedural guidelines for standardization of patient preparation, scan acquisition, and image processing to ensure the most accurate and reproducible quantitative PET measurements $(11,12)$. In brief, patients fasted $6 \mathrm{~h}$ prior to the radiotracer injection (target serum glucose $\leq 150 \mathrm{mg} / \mathrm{dL}$ ). A static whole-body (skull to mid-thigh) PET scan was started 60 min (range of 55-75 min) after injection of ${ }^{18} \mathrm{~F}-\mathrm{FDG}(3-4 \mathrm{Mbq} / \mathrm{kg}$ ), with an acquisition time of 90 s per bed position. A low-dose CT was performed prior to the PET scan. All PET data were normalized and corrected for scatter and random events, attenuation and decay.

Quality assessment for patient preparation, imaging protocols and anonymization for central review of PET/CT images were ensured by an independent dedicated academic PET/CT imaging core lab (ORILaB). Items checked in the quality control analysis were already described in a previous report and this quality control was applied to all ${ }^{18} \mathrm{~F}-\mathrm{FDG}$ PET/CT scans of the current study (2). Any violation with respect to: uptake time, administered dose, complete image data set, good quality of images (high statistics suitable for diagnostic interpretation), PET/CT scans of the same patient performed on the same scanner for baseline and early time-points, and time between baseline PET/CT and treatment start for all ${ }^{18} \mathrm{~F}-\mathrm{FDG}$ PET/CT scans of this study led to the exclusion from the central review analysis. None of the nuclear medicine physicians involved in this study had access to the medical records and treatment outcomes. Those were centralized and stored in the data center. All PET measurements were computed on a dedicated 
workstation (Advantage Workstation; GE Healthcare) using the commercial PETVCAR software, version 4.6 (GE Healthcare).

Target lesions identified for each patient were defined as follows: unequivocal tumor origin, transverse diameter greater than $15 \mathrm{~mm}$ on a registered CT image, and an ${ }^{18} \mathrm{~F}-\mathrm{FDG}$ SUV normalized to lean body mass (SUL) higher than $1.5 \times$ the mean liver SUL $+2 \times \mathrm{SD}$, or in the presence of liver metastasis, $2.0 \times$ mean aorta SUL $+2 \times \mathrm{SD}$, following PERCIST methodology (13). In case there was no target lesion identified on the baseline PET/CT, the patient was excluded from the baseline WB-MATV and from the response analysis.

The image analysis procedure for the different PET metrics used in this study was as follows: the MATV of a lesion was defined as the volume of tumor tissue demonstrating metabolic activity at or higher than the calculated PERCIST threshold described above. Baseline WB-MATV was calculated as the sum of the MATV values of all target lesions, without a predefined limitation on their number. To minimize overestimation of WB-MATV, volume of interest for each lesion was manually placed so as to exclude both surrounding physiological uptake and adjacent lesions' uptake.

Different response criteria were used for the evaluation of the early mR: PERCIST-30\%, PERCIST with an adapted response threshold of 15\%, EORTC (European Organisation for Research and Treatment of Cancer)-15\%, and CONSIST-15\% $(5,13,14)$.

For all these response criteria, the early $\mathrm{mR}$ assessment was dichotomized into metabolic responder $(\mathrm{mR})$ and non-responder $(\mathrm{mNR})$. With CONSIST methodology, a patient was classified as non-responder when there was at least one target lesion not reaching a SULmax decrease of $>15 \%(5,15)$. With PERCIST and EORTC methodologies, patients who had a 
complete or partial metabolic response were classified as $\mathrm{mR}$ and patients who had a stable or progressive metabolic disease were classified as mNR. More details on criteria used in this study for the different metabolic response assessment methodologies can be found in the Supplemental Table 1.

All PET measurements were normalized to lean body mass except for EORTC measurements which were normalized to body surface area as required in the guidelines (14).

\section{Statistical Analysis}

The baseline clinical characteristics and survival data were collected prospectively. For univariable analyses, survival outcomes were measured from the date of treatment start to death from any cause for OS, and to the point of tumor progression or recurrence (based on radiological assessment according to RECIST 1.1 with either contrast-enhanced CT-scan or MRI which was done at baseline and every 2 cycles ( 8 weeks)) or death from any cause for PFS. For univariable and multivariable analyses of the early $\mathrm{mR}$ assessment, survival outcomes were measured from the date of the early $\mathrm{mR}$ assessment to death from any cause for OS and to the point of progression or recurrence (according to RECIST 1.1 evaluation which was done every 2 cycles) or death from any cause for PFS. All patients alive or not progressing at last follow-up were censored.

As the optimal cutoff value for baseline WB-MATV was determined and validated in a recent report to be $100 \mathrm{~cm}^{3}$ in chemorefractory $\mathrm{mCRC}$ patients, the same cutoff was applied in the external validation set (2).

The prognostic values of the clinical and PET parameters (baseline WB-MATV and early $\mathrm{mR}$ ) were assessed using Kaplan-Meier estimation for survival probabilities (OS and PFS), the 
log-rank test for comparisons of groups, and the Cox proportional hazards regression model for regression analysis to estimate the hazard ratios (HR) with $95 \%$ confidence intervals (CI). In the multivariable Cox model, the following variables were considered for association with OS and PFS: age, gender, body mass index, Eastern Cooperative Oncology Group performance status, KRAS mutational status, primary tumor location (right- versus left-sided colon and rectum), baseline WB-MATV, and early $\mathrm{mR}$ following response criteria as described above. BRAF mutational status was only included in the statistical analyses of the validation set due to the small number of BRAF mutant patients remaining in last-line of treatment.

The predictive accuracy for OS and PFS of the different early $\mathrm{mR}$ methods was assessed by the Harrell's c-index. $P$ values of $<0.05$ were considered statistically significant, and all tests were two-sided. Statistical analyses were carried out using SAS, version 9.4 (SAS Institute), IBM SPSS Statistics 25 (IBM Corporation, Armonk, NY) and GraphPad Prism, version 7.04 (GraphPad Software Inc.).

\section{RESULTS}

\section{Patients}

Out of 239 mCRC patients included in the Belgian cohort and 125 in the Italian cohort, $224(94 \%)$ and $109(87 \%)$ respectively were considered suitable for baseline WB-MATV analysis, while $192(80 \%)$ and $94(75 \%)$ patients were retained for early $\mathrm{mR}$ analysis. The reasons for ineligibility are shown in the study flow diagram in Figure 1. Patient and disease characteristics are summarized in Supplemental Table 2.

The median durations of follow-up were respectively 24.0 months and 25.1 months for the development and the validation sets. At the end of the studies of the development and external 
validation sets, 217/224 (97\%) and 87/109 (80\%) patients had died, respectively and all patients had a progression event. Median OS and PFS for all patients eligible for analysis were 6.9 mo (95\% CI, 6.2-8.1 mo) and $3.3 \mathrm{mo}(95 \% \mathrm{CI}, 2.2-3.7 \mathrm{mo})$ respectively for the development set and $25.2 \mathrm{mo}$ (range, 20.9-27.2 mo) and $9.7 \mathrm{mo}(95 \% \mathrm{CI}, 8.4-11.5 \mathrm{mo})$ respectively for the validation set.

\section{Baseline clinical factors and Patient Outcomes}

Among the clinical factors, the following were found to be statistically significant for OS in the development set: ECOG PS (HR: $1.59(1.21-2.09), P=0.001)$ and BMI (HR: 0.57 (0.43$0.76), P<0.001)$ and for OS in the validation set: BRAF mutational status (HR: 3.43 (1.1110.54), $P=0.03)$ and ECOG PS (HR: $1.97(1.06-3.69), P=0.03)$.

\section{Baseline WB-MATV}

The median values for baseline WB-MATV in the development and validation sets were $164 \mathrm{~cm}^{3}$ (5th-95th percentiles, 6-1755 $\mathrm{cm}^{3}$ ), and $134 \mathrm{~cm}^{3}$ (5th-95th percentiles, 6-1426 $\mathrm{cm}^{3}$ ), respectively.

The median values of the number of weeks that have passed between the baseline PET to the start of treatment in the development and validation sets were 1 (range, $0-4$ ), and 1 (range, 0 6), respectively.

\section{Baseline WB-MATV and Patient Outcomes}

In the development set, patients with a high baseline WB-MATV $\left(\geq 100 \mathrm{~cm}^{3}\right)$ had a significantly worse outcome compared to patients with a low baseline WB-MATV $\left(<100 \mathrm{~cm}^{3}\right)$ both in terms of median OS (4.5 months (95\% CI, 3.4-5.5) vs 11.2 months (95\% CI, 9.4-13.9); 
HR: $2.70, P<0.001)$ and median PFS $(1.9$ months (95\% CI, 3.5-5.7) vs 4.3 months (95\% CI, 9.4-13.9); HR: 1.98, $P<0.001)$.

These results were confirmed in the validation set: patients with a high baseline WBMATV had a significantly worse outcome compared to patients with a low baseline WB-MATV both in terms of median OS (20.9 months (95\% CI, 17.2-24.6) vs 35.7 months (95\% CI, 22.249.1); HR: 1.93, $P=0.003$ ) and median PFS (9.1 months (95\% CI, 7.4-10.7) vs 12.4 months (95\% CI, 9.0-15.9); HR: 1.86, $P=0.002)$ (Figure 2A, 2B and Table 1).

\section{Early mR Following Different Response Criteria and Patient Outcomes}

All $\mathrm{mR}$ methods applied at an early time-point (PERCIST-15\%, EORTC, and CONSIST), except for PERCIST-30\%, have shown to be highly predictive of OS and PFS in both the development and validation sets (Figure 3A, 3B and Table 1).

In terms of diagnostic performance, the early $\mathrm{mR}$ assessment according to the CONSIST criteria was found to be the most predictive method for both OS and PFS in the development and validation sets (Supplemental Table 3). The median values of the number of target lesions per patient evaluated with the CONSIST method in the development and validation sets were 4 (range, 1-35), and 3 (range, 1-21), respectively.

As early $\mathrm{mR}$ with PERCIST-30\% was not found to be predictive of PFS in the development set and of OS and PFS in the validation set, this method was only included in the multivariable analyses of OS in the development set.

PET images with examples of patients showing low/high WB-MATV associated with response/non-response are illustrated in Figure 4. Example of a patient subject to differences in 
response assessment following PERCIST and EORTC methodologies is shown in Supplemental Figure 1.

\section{Independent Predictors of OS and PFS Among PET and Clinical Parameters}

After adjustment for clinical parameters, the multivariable analyses identified baseline WB-MATV as a significant independent predictor of OS (HR: 2.56 and 1.87, $P<0.001$ and $P=$ 0.005, for the development and validation sets, respectively) and PFS (HR: 2.0 and 1.94, $P<$ 0.001 and $P=0.002$ ) (Table 2).

After adjustment for clinical parameters and baseline WB-MATV, early mR according to CONSIST was identified as a significant independent predictor of OS (HR: 1.55 and 1.79, $P=$ 0.005 and $P=0.02$ ) and PFS (HR: 1.64 and 1.69, $P<0.001$ and $P=0.03$ ) (Table 2).

\section{Combining Baseline WB-MATV and Early mR Assessment}

Combining baseline WB-MATV and early $\mathrm{mR}$ according to CONSIST classified the patients into four categories. Survival graphs of these four risk groups in the development and validation sets for both OS and PFS are shown in Figure 5. 


\section{DISCUSSION}

This study is the first to prospectively validate baseline whole-body metabolically active tumor volume (WB-MATV) and early metabolic response assessment (mR) as strong ${ }^{18} \mathrm{~F}-\mathrm{FDG}$ PET/CT-based biomarkers in both chemonaïve (treated with standard first-line chemotherapy combined with targeted agents) and chemorefractory (treated with targeted agents) mCRC patients. This study showed that baseline WB-MATV and early $\mathrm{mR}$ performed after one treatment cycle (i.e. at 2 weeks) were able to identify a subset of high-risk patients. These highrisk patients (high WB-MATV and metabolic non-responders (mNR)) had a risk of experiencing disease progression or dying 3 times higher than low-risk patients (low WB-MATV and metabolic responders $(\mathrm{mR})$ ). The predictive value of early $\mathrm{mR}$ was demonstrated to be independent of baseline WB-MATV and clinical factors in the two clinical settings. Moreover, combining WB-MATV and early $\mathrm{mR}$ allowed a better risk stratification in identifying distinct patient risk groups in first or last-line of treatment.

Our study confirmed the added prognostic value of baseline WB-MATV beyond the usual clinical prognostic parameters for both OS and PFS in chemonaïve patients. To the best of our knowledge, this is the first report that investigated baseline WB-MATV as prognostic biomarker in first-line setting. Our results have shown that baseline WB-MATV is predictive of survival regardless of treatment administered and, therefore, can be considered as a pure prognostic biomarker (16).

In addition to the validation of WB-MATV as a baseline stratification factor in mCRC in first-line setting, another important contribution of this study is that it highlighted the predictive value of early metabolic response assessment for OS/PFS in both first and last-line treatment settings. The predictive values of early $\mathrm{mR}$ in the first-line were almost the same as those 
obtained in the last-line setting and in line with those reported in small case series, which were conducted without clinical validation $(5,6,17,18)$. Conversely, a few studies investigating mCRC patients reported a lack of correlation between early $\mathrm{mR}$ and outcomes, but those had several methodological limitations $(19,20)$. In particular the study of Byström et al. lacked basic conditions of imaging standardization and quality control. The results of our prospective validation study strongly contradicts the conclusion made by Byström et al.'s that "routine monitoring of mCRC patients by PET scans is not recommended due to its too limited clinical value and notably in first-line treatment setting" (19).

Several $\mathrm{mR}$ methods applying different criteria were also investigated in this study. Our findings indicate that the clinical impact of using a $\mathrm{mR}$ method or another is minimal in terms of outcome prediction, except for PERCIST.

PERCIST-30\% applied in the context of early $\mathrm{mR}$ assessment was not predictive of outcomes in both first and last-line treatment settings, except for OS in last-line. Conversely, PERCIST-15\% was found to be a strong predictor of outcomes in both first and last-line treatment settings. These results suggest that the PERCIST method with the response threshold set at $30 \%$ for a response assessment usually performed after 3-4 cycles of therapy has to be adapted in an early response setting with a threshold set at $15 \%$.

Interestingly, the CONSIST method, based on the hypothesis that treatment-resistant emergent clones are reflected by lesions which do not significantly decrease their metabolism under treatment, was shown to have the highest predictive value for OS/PFS. This method, when applying a response threshold of $15 \%$, was previously reported by our group to have a high negative predictive value (95\%) (5). As this response threshold (15\%) was also applied in this study to the adapted PERCIST-15\% and EORTC and those did not demonstrate a predictive 
value of outcomes as high as the CONSIST method, the criteria used in this methodology could explain its higher predictive value.

Another major finding of this study in addition to the validation of baseline WB-MATV and early $\mathrm{mR}$ as strong predictive biomarkers independently of treatment lines, is that the added predictive value of early $\mathrm{mR}$ when combined with WB-MATV strongly depends on the baseline tumor load and the treatment-line.

In low baseline WB-MATV patients in last-line of treatment, where OS is the most important endpoint, the combination of the two biomarkers has enabled the identification of two risk groups of patients with significantly distinct median OS: responders vs non-responders. A trend, due to the limited number of patients included in the low WB-MATV and non-responders group $(\mathrm{n}=$ 10), was also found in low baseline WB-MATV patients in first-line of treatment for PFS, as in this setting PFS is the relevant endpoint when a treatment change may be considered. In both settings, for the group of responder patients with low baseline WB-MATV, the prognostic information provided could reinforce the oncologist in his therapeutic decisions. In the group of non-responder patients with low baseline WB-MATV, the rapid identification of a limited number of non-responding lesions (oligo-resistance) could lead to treatment adaptation by adding locoregional ablative treatments centered on the PET-resistant lesions. If metabolic treatment resistance is observed in the majority of lesions, rapid shift to an alternative treatment regimen or referral to an appropriate clinical trial could be considered. In patients showing clinical or biological signs of intolerance, the absence of a metabolic response can be an additional argument for deciding an early treatment adaptation before radiological progression is documented. Our findings therefore support the clinical use of early $\mathrm{mR}$ to discriminate the level of risk of low baseline WB-MATV mCRC patients across all treatment lines. 
For high baseline WB-MATV patients in both treatment lines, the fact that they are responders or non-responders does not significantly affect their outcomes. This suggests that performing an early $\mathrm{mR}$ in these high tumor load patients is probably not useful. Several factors may explain these results. Firstly, the low metabolic response threshold (minimum 15\% SULmax decrease) used by the CONSIST method maximizes the negative predictive value to avoid eliminating a potentially efficient treatment. This low threshold also minimizes the positive predictive value, impairing any distinction on the depth of response. Secondly, for high baseline WB-MATV patients, the lack of randomized control group precludes knowing whether responders have a survival benefit over untreated patients. Therefore, we can only state that performing an early $\mathrm{mR}$ may not be useful in these high tumor load patients but we should in no way extrapolate from this finding that treatments are not effective.

A potential limitation of this study is that the population of the development set was already used in a previous study assessing the prognostic value of baseline WB-MATV (population split in two sets for internal validation) (2).

In terms of perspectives, PET-driven treatment escalation strategies for high-risk patients, identified at an early time-point, might be effective to prolong survival. Further studies would be needed to assess the impact of these adaptive treatment strategies on survival outcomes. 


\section{CONCLUSION}

This study validates baseline WB-MATV and early $\mathrm{mR}$ as strong independent prognostic biomarkers for OS/PFS in first and last-line mCRC treatment settings - stronger than the relevant usual clinical parameters. Combining these two biomarkers significantly increased the overall prognostic accuracy and allowed a better risk stratification in identifying distinct risk groups of patients with significant different median OS and PFS in first and last-line treatment settings. Therefore, the use of these two biomarkers could be proposed as stratification factors in clinical trials. Their use could also be recommended in clinical oncology for risk-stratification in mCRC patients. 


\section{DISCLOSURE}

Francesco Sclafani - Consultancy, advisory board: Bayer. Research funding: AstraZeneca, Bayer, BMS, Roche. Travel grants: Bayer, Lilly. Alain Hendlisz - Consultancy, advisory board: Amgen, Bayer, Eli Lilly, Merck, Pierre Fabre, Servier, Sirtex. Research funding: Amgen, AstraZeneca, Ipsen, Leo Pharma, Merck, Roche, Sanofi, Teva Pharma. Travel grant: Merck, Roche, Sirtex. All remaining authors have declared no conflicts of interests. 


\section{KEY POINTS}

\section{QUESTION:}

Does early metabolic response yield additional prognostic value compared to baseline clinical parameters and WB-MATV in mCRC patients under first or last-line of treatment?

\section{PERTINENT FINDINGS:}

This study, including three prospective trials ( 2 development and 1 external validation datasets), validates baseline WB-MATV and early metabolic response as independent prognostic biomarkers for OS/PFS in mCRC, independently of patients' treatment line. The added prognostic value of early metabolic response assessment was found mostly in those patients with low baseline WB-MATV.

\section{IMPLICATIONS FOR PATIENT CARE:}

Combining these two PET biomarkers should be implemented in future clinical trials and in clinical routine for monitoring $\mathrm{mCRC}$ patients under first or last-line of treatment. 


\section{REFERENCES}

1. Siegel RL, Miller KD, Jemal A. Cancer statistics, 2019. CA Cancer J Clin. 2019;69:7-34.

2. Woff E, Hendlisz A, Ameye L, et al. Validation of metabolically active tumor volume and total lesion glycolysis as 18 F-FDG PET/CT-derived prognostic biomarkers in chemorefractory metastatic colorectal cancer. $J$ Nucl Med. 2019;60:178-184.

3. Hendlisz A, Golfinopoulos V, Garcia C, et al. Serial FDG-PET/CT for early outcome prediction in patients with metastatic colorectal cancer undergoing chemotherapy. Ann Oncol. 2012;23:1687-1693.

4. Gebhart G, Gámez C, Holmes E, et al. ${ }^{18}$ F-FDG PET/CT for early prediction of response to neoadjuvant Lapatinib, Trastuzumab, and their combination in HER2-positive breast cancer: Results from Neo-ALTTO. J Nucl Med. 2013;54:1862.

5. Woff E, Hendlisz A, Garcia C, et al. Monitoring metabolic response using FDG PET-CT during targeted therapy for metastatic colorectal cancer. Eur J Nucl Med Mol Imaging. 2016;43:1792-1801.

6. Skougaard K, Nielsen D, Jensen BV, Hendel HW. Comparison of EORTC criteria and PERCIST for PET/CT response evaluation of patients with metastatic colorectal cancer treated with Irinotecan and Cetuximab. J Nucl Med. 2013;54:1026-1031.

7. Kim JH. Comparison of the EORTC criteria and PERCIST in solid tumors: a pooled analysis and review. Oncotarget. 2016;7:58105-58110.

8. Cremolini C, Schirripa M, Antoniotti C, et al. First-line chemotherapy for mCRC-a review and evidence-based algorithm. Nat Rev Clin Oncol. 2015;12:607-619.

9. Deleporte A, Hendlisz A, Garcia C, et al. SoMore trial: Early metabolic response assessment of a sorafenib (SOR) and capecitabine (CAP) combination in chemorefractory metastatic colorectal cancer (mCRC). J Clin Oncol. 2014;32:524-524.

10. Hendlisz A, Deleporte A, Vandeputte $\mathrm{C}$, et al. Regorafenib assessment in refractory advanced colorectal cancer: RegARd-C study protocol. BMJ Open. 2015;5:e07189e007189.

11. Boellaard R, Delgado-Bolton R, Oyen WJG, et al. FDG PET/CT: EANM procedure guidelines for tumour imaging: version 2.0. Eur J Nucl Med Mol Imaging. 2015;42:328354.

12. Aide N, Lasnon C, Veit-Haibach P, Sera T, Sattler B, Boellaard R. EANM/EARL harmonization strategies in PET quantification: from daily practice to multicentre oncological studies. Eur J Nucl Med Mol Imaging. 2017;44:17-31. 
13. Wahl RL, Jacene H, Kasamon Y, Lodge MA. From RECIST to PERCIST: evolving considerations for PET Response Criteria in Solid Tumors. J Nucl Med. 2009;50:122S$150 \mathrm{~S}$.

14. Young H, Baum R, Cremerius U, et al. Measurement of clinical and subclinical tumour response using [18F]-Fluorodeoxyglucose and Positron Emission Tomography: review and 1999 EORTC recommendations. :10.

15. Hendlisz A, Deleporte A, Delaunoit $\mathrm{T}$, et al. The prognostic significance of metabolic response heterogeneity in metastatic colorectal cancer. Santini D, ed. PLOS ONE. 2015; 10:e0138341.

16. Ballman KV. Biomarker: Predictive or prognostic? J Clin Oncol. 2015;33:3968-3971.

17. Lim Y, Bang J-I, Han S-W, et al. Total lesion glycolysis (TLG) as an imaging biomarker in metastatic colorectal cancer patients treated with regorafenib. Eur J Nucl Med Mol Imaging. 2017;44:757-764.

18. de Geus-Oei L-F, Vriens D, van Laarhoven HWM, van der Graaf WTA, Oyen WJG. Monitoring and predicting response to therapy with 18F-FDG PET in colorectal cancer: a systematic review. J Nucl Med. 2009;50:43S-54S.

19. Bystrom P, Berglund A, Garske U, et al. Early prediction of response to first-line chemotherapy by sequential [18F]-2-fluoro-2-deoxy-D-glucose positron emission tomography in patients with advanced colorectal cancer. Ann Oncol. 2009;20:1057-1061.

20. Choi M, Kollepara SLS, Heilbrun LK, Smith D, Shields AF, Philip PA. PET Scans as a predictive marker of survival in advanced colorectal cancer. Clin Colorectal Cancer. 2015; $14: 35-40$. 


\section{FIGURES}

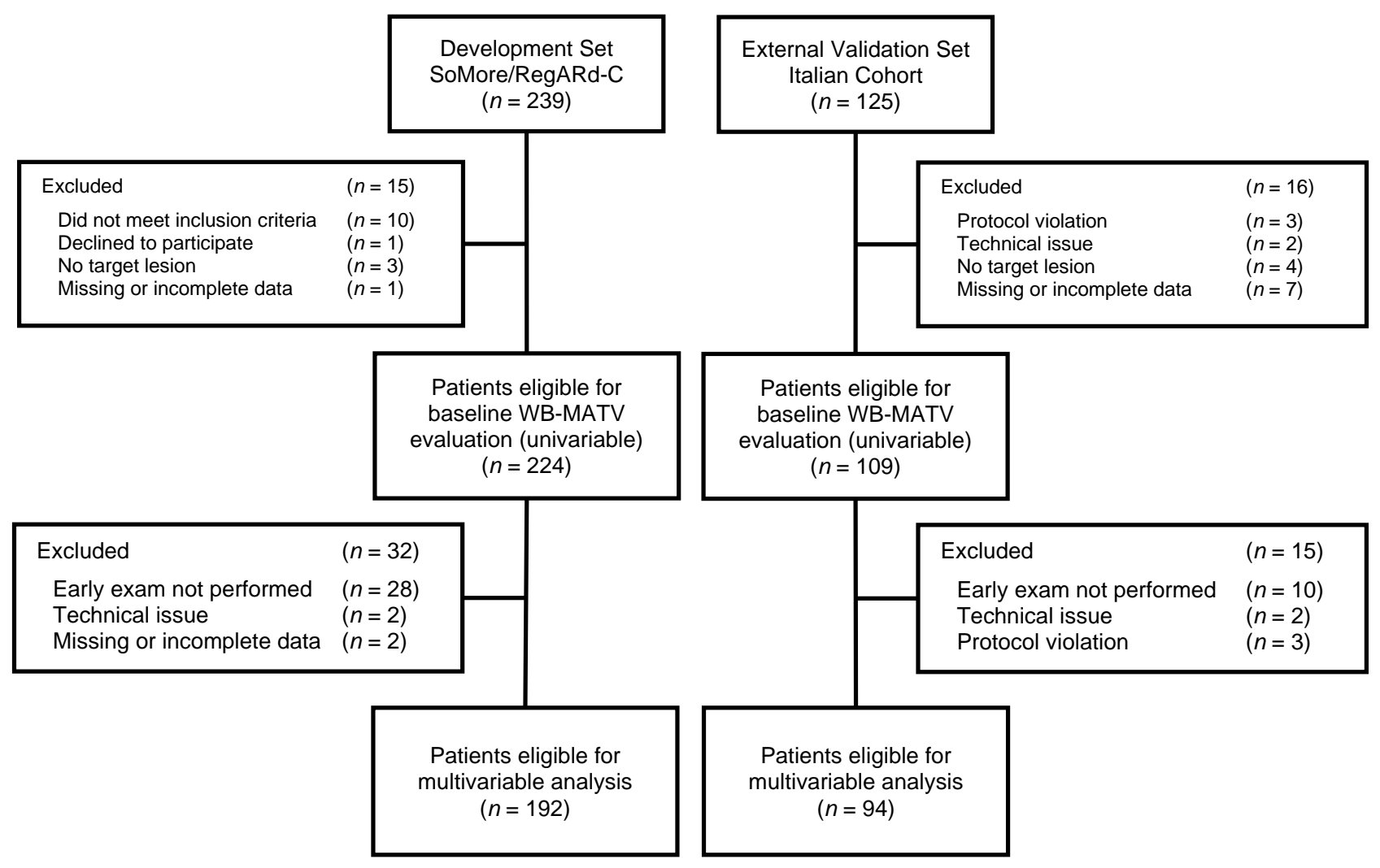

FIGURE 1. Study flow diagram of the development and external validation sets. 
A

\section{OS baseline WB-MATV}

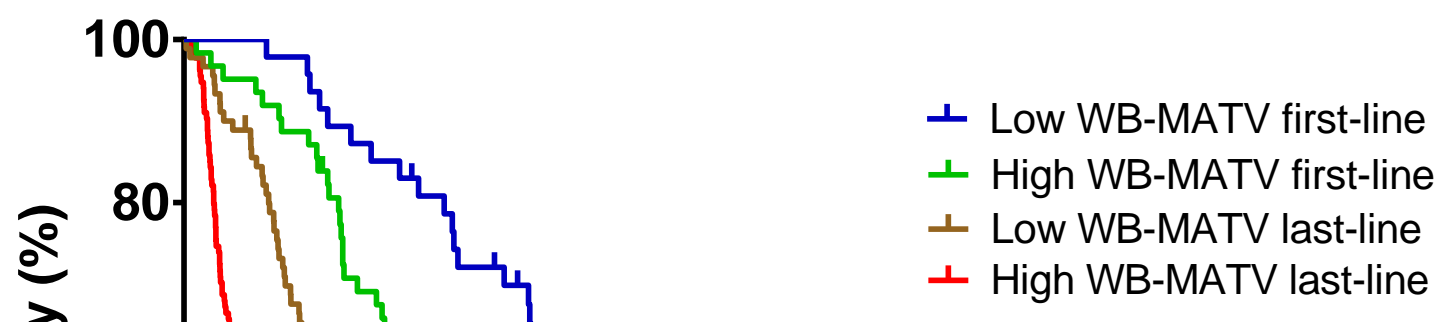

Number of subjects at risk

Time (mo)

Low WB-MATV first-line

High WB-MATV first-line Low WB-MATV last-line High WB-MATV last-line
601 HR first-line: $1.93(95 \% \mathrm{Cl}, 1.26-2.97)$ $P=0.003$

HR last-line: $2.70(95 \% \mathrm{Cl}, 2.02-3.62)$ $P<0.001$

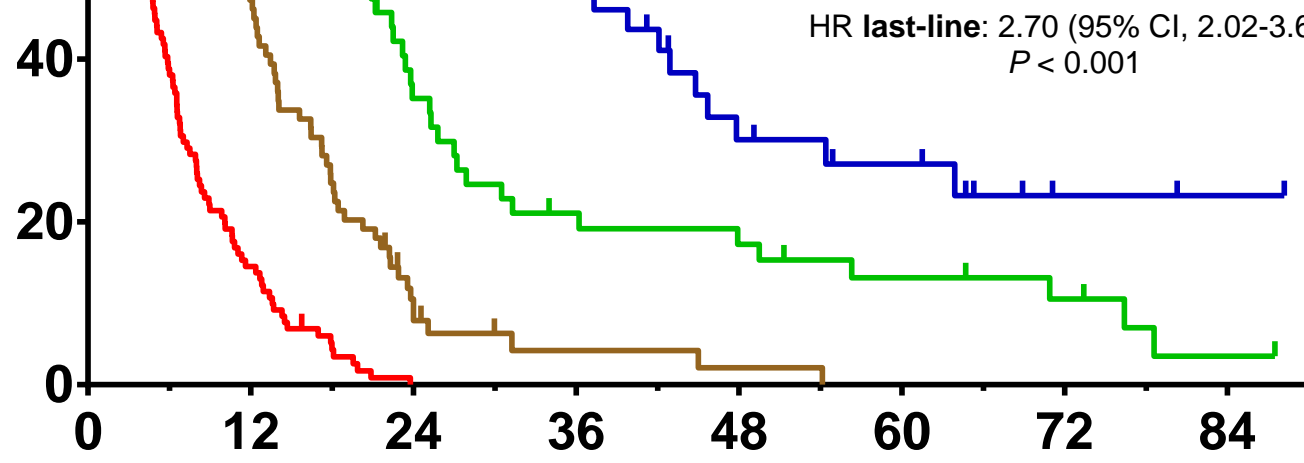


B $\quad$ PFS baseline WB-MATV

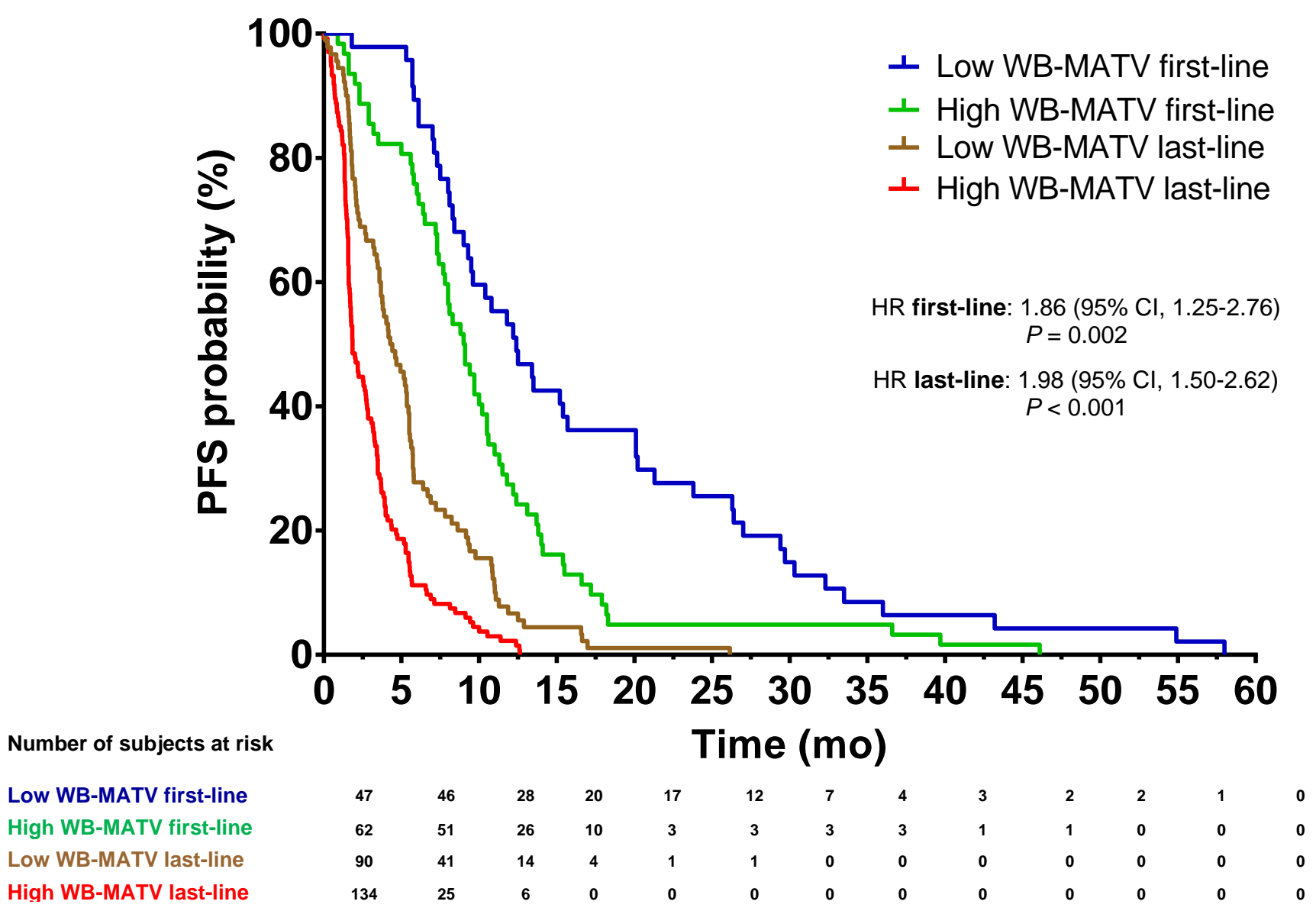

FIGURE 2. Kaplan-Meier estimates of OS (A) and PFS (B) according to baseline WB-MATV in the development set (last-line) and validation set (first-line). 
A

\section{OS early metabolic response}

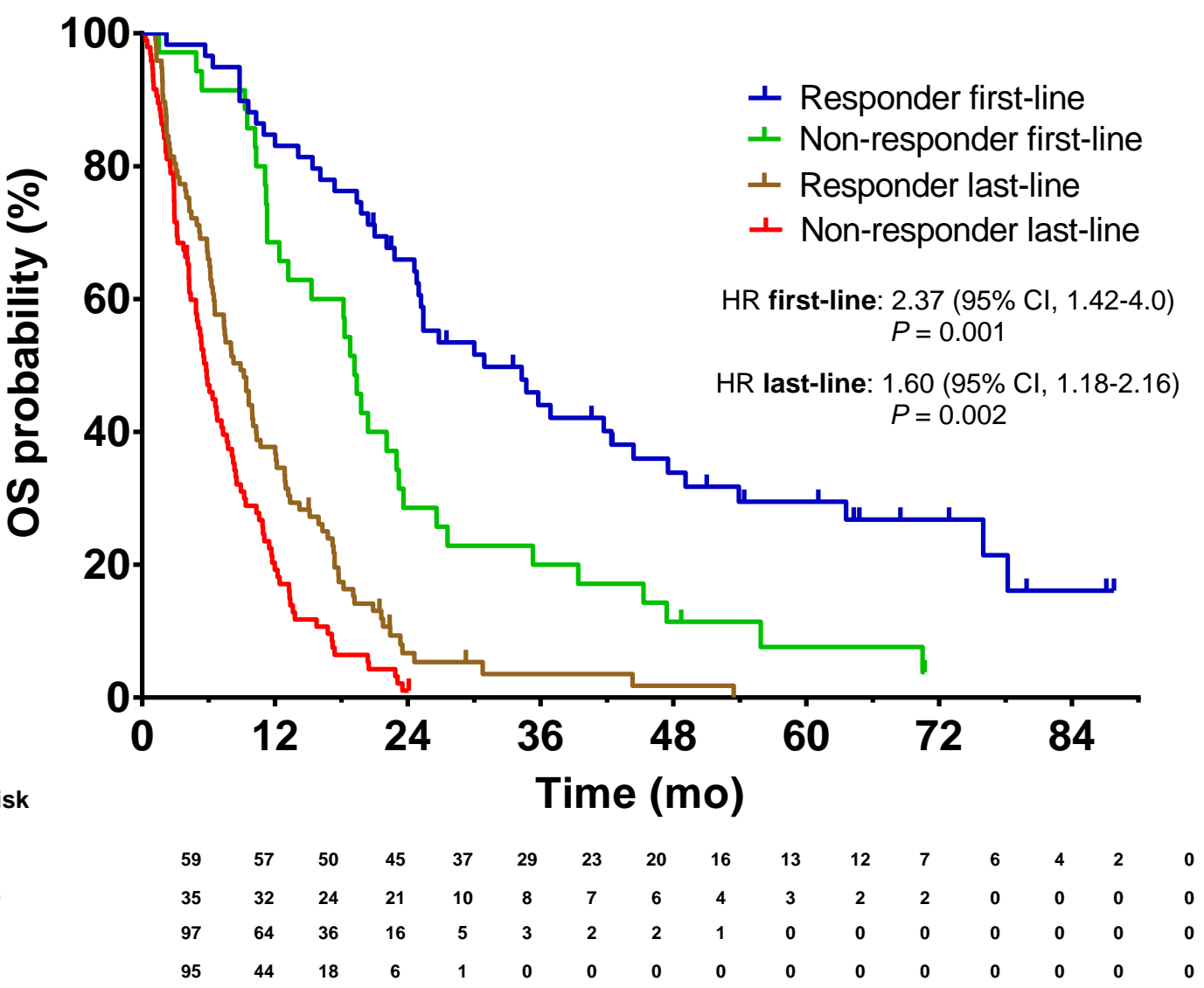


B

\section{PFS early metabolic response}

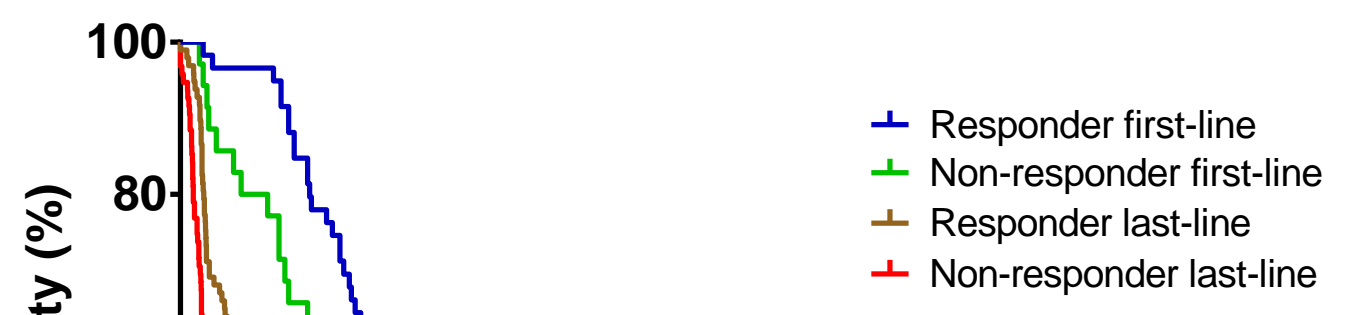

HR first-line: $2.16(95 \% \mathrm{Cl}, 1.33-3.51)$

$P=0.002$

HR last-line: $1.86(95 \% \mathrm{Cl}, 1.37-2.52)$ $P<0.001$

Number of subjects at risk Time (mo)

\section{Responder first-line}

Non-responder first-line

Responder last-line

Non-responder last-line

$\begin{array}{lcccccccccccc}59 & 56 & 37 & 20 & 14 & 13 & 7 & 6 & 4 & 3 & 2 & 1 & 0 \\ 35 & 27 & 11 & 7 & 2 & 2 & 2 & 1 & 0 & 0 & 0 & 0 & 0 \\ 97 & 32 & 12 & 3 & 1 & 1 & 0 & 0 & 0 & 0 & 0 & 0 & 0 \\ 95 & 15 & 4 & 0 & 0 & 0 & 0 & 0 & 0 & 0 & 0 & 0 & 0\end{array}$

FIGURE 3. Kaplan-Meier estimates of OS (A) and PFS (B) according to early $m R$ using CONSIST method in the development set (last-line) and validation set (first-line). 


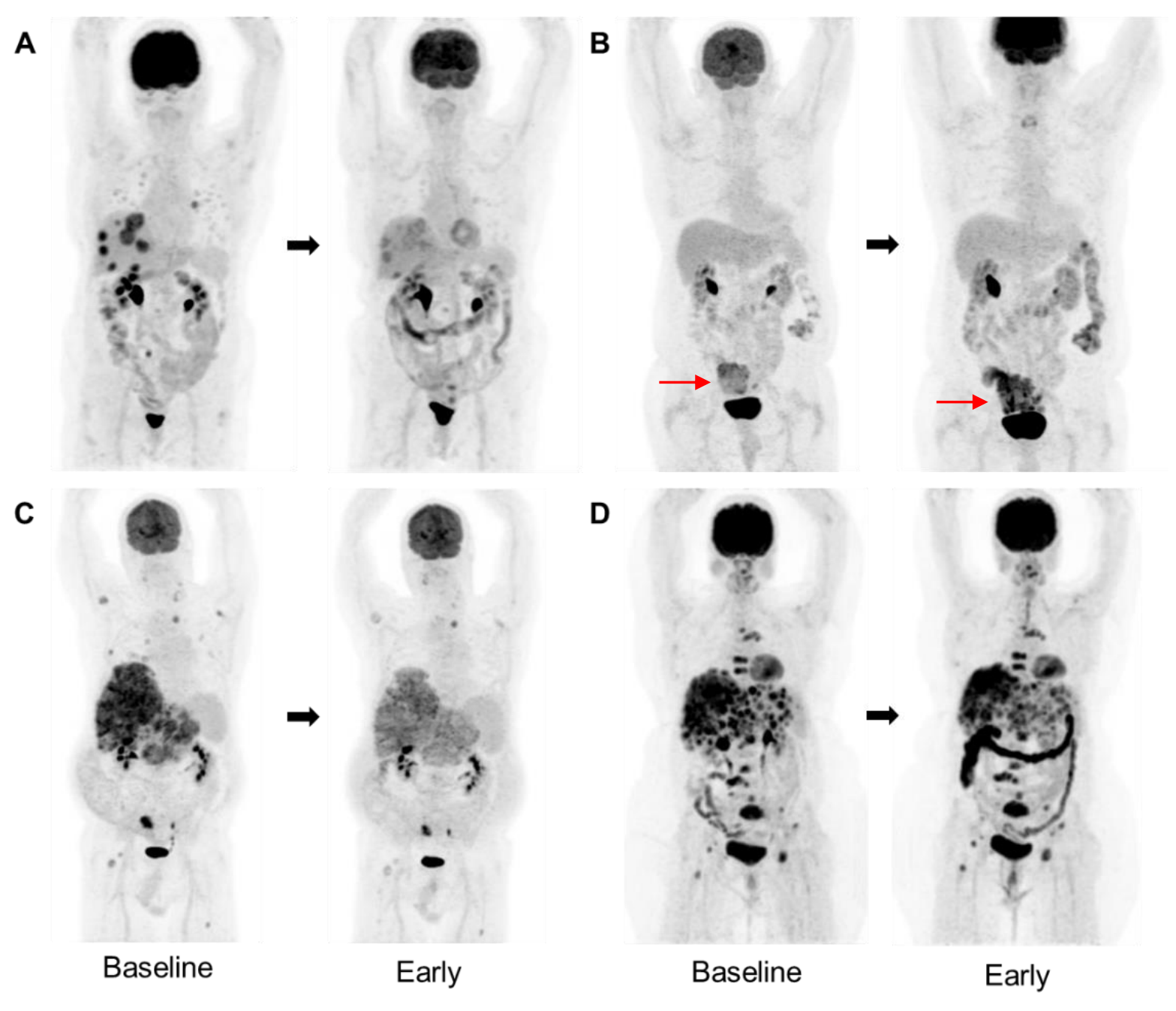

FIGURE 4. Examples of PET maximum-intensity projections images of patients at baseline and early time-points with a low baseline WB-MATV $\left(85 \mathrm{~cm}^{3}\right)$ who respond (A), with a low baseline WB-MATV $\left(30 \mathrm{~cm}^{3}\right.$ ) who did not respond (resistant lesion showed by red arrows) (B), with a high baseline WB-MATV $\left(2336 \mathrm{~cm}^{3}\right)$ who respond $(\mathrm{C})$, and with a high baseline WB-MATV $\left(1065 \mathrm{~cm}^{3}\right)$ who did not respond (multiple resistant lesions) (D). 
A

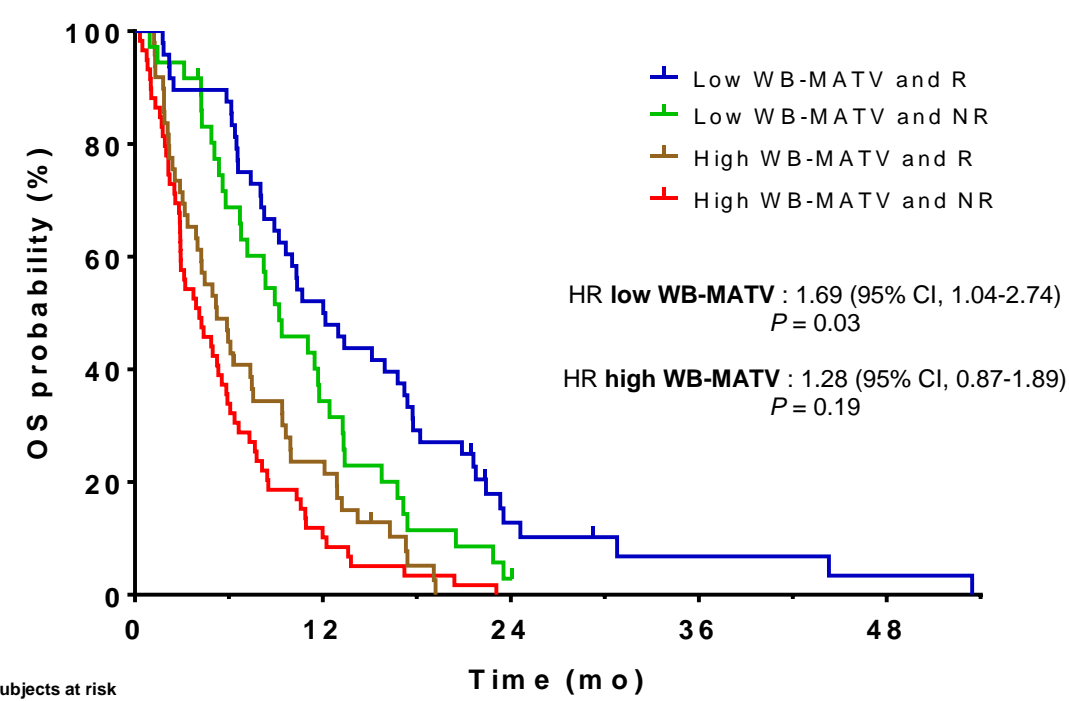

Number of subjects at risk

Low WB-MATV and R

Low WB-MATV and NR
High WB-MATV and $R$

High WB-MATV and R
High WB-MATV and NR

Time (mo)
B

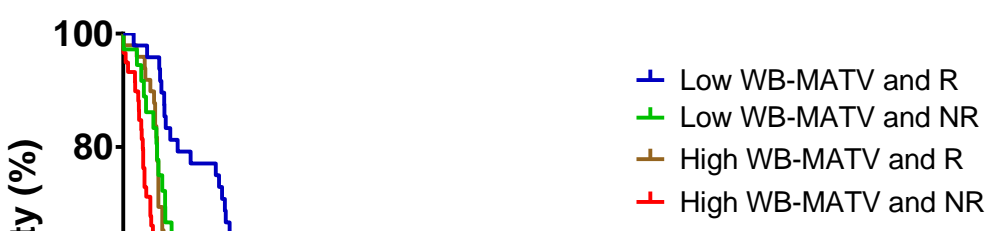

HR low WB-MATV : $1.94(95 \% \mathrm{Cl}, 1.2-3.13)$ $P=0.007$

HR high WB-MATV : $1.64(95 \% \mathrm{Cl}, 1.11-2.43)$ $P=0.01$

Time (mo)

20

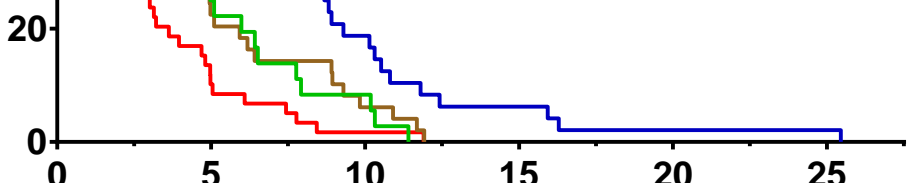

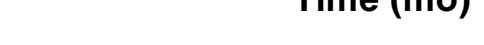

$\begin{array}{llllllllllll}48 & 37 & 21 & 13 & 9 & 3 & 3 & 1 & 1 & 1 & 1 & 0 \\ 36 & 19 & 9 & 5 & 3 & 0 & 0 & 0 & 0 & 0 & 0 & 0 \\ 49 & 24 & 11 & 7 & 3 & 0 & 0 & 0 & 0 & 0 & 0 & 0 \\ 59 & 19 & 6 & 3 & 1 & 0 & 0 & 0 & 0 & 0 & 0 & 0\end{array}$ 


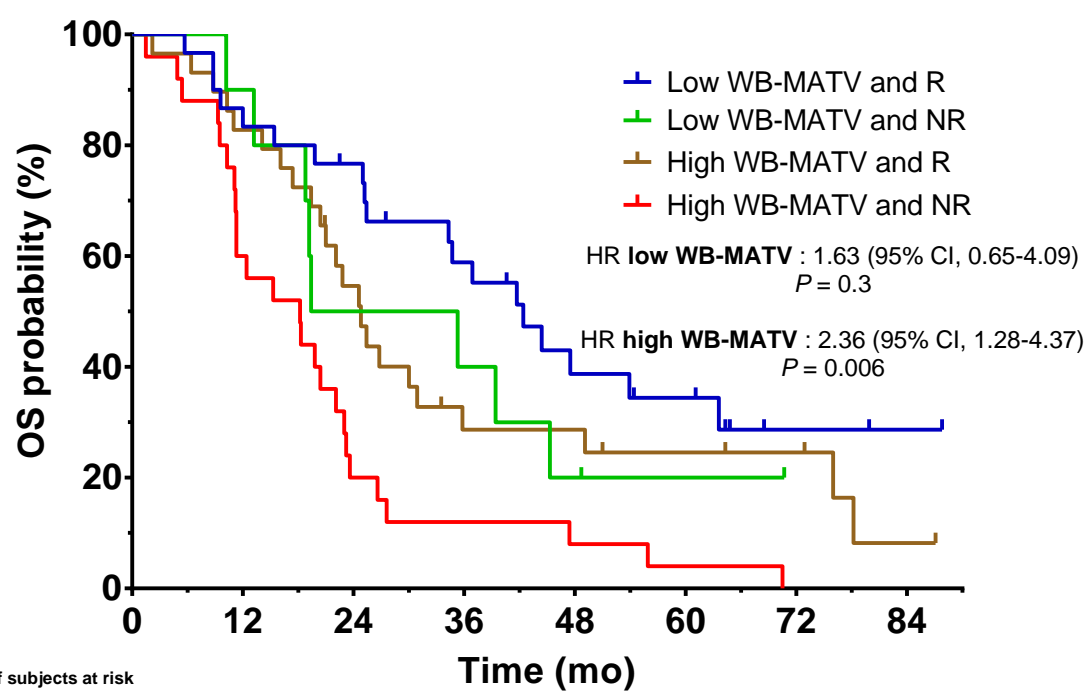

Low WB-MATV and $R$

Low WB-MATV and

High WB-MATV and $A$
High WB-MATV and

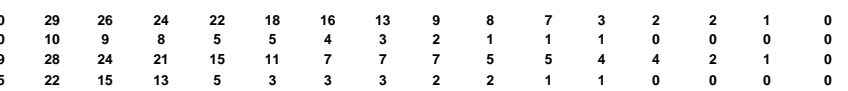

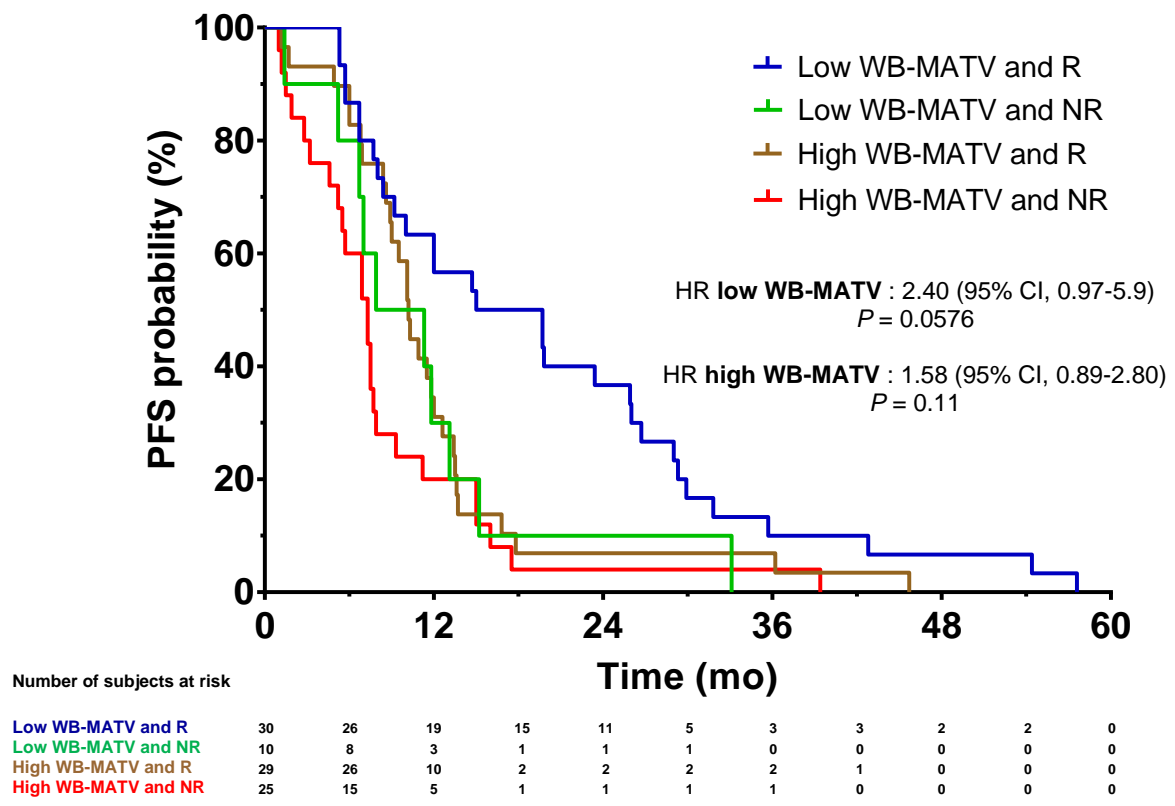

FIGURE 5. Kaplan-Meier estimates of OS and PFS according to baseline WB-MATV combined with early mR using CONSIST method in the development set (A and B) and validation set (C and D) classifying patients into four risk groups. 


\section{TABLES}

TABLE 1. Univariable Analyses of Baseline WB-MATV and Early Metabolic Response According to Different Methods for OS and PFS in Development and Validation Sets.

\begin{tabular}{|c|c|c|c|c|c|c|c|c|}
\hline \multirow{4}{*}{ Variables } & \multicolumn{4}{|c|}{ Development Set } & \multicolumn{4}{|c|}{ Validation Set } \\
\hline & OS & & PFS & & OS & & PFS & \\
\hline & \multicolumn{4}{|c|}{ Univariable analysis } & \multicolumn{4}{|c|}{ Univariable analysis } \\
\hline & $\mathrm{HR}(95 \% \mathrm{CI})$ & $P$ & HR $(95 \%$ CI $)$ & $P$ & HR $(95 \% \mathrm{CI})$ & $P$ & HR $(95 \% \mathrm{CI})$ & $P$ \\
\hline $\begin{array}{l}\text { Baseline WB-MATV } \\
\text { Early Metabolic } \\
\text { Response according to: }\end{array}$ & $2.70(2.02-3.62)$ & $<0.001$ & $1.98(1.50-2.62)$ & $<0.001$ & $1.93(1.26-2.97)$ & 0.003 & $1.86(1.25-2.76)$ & 0.002 \\
\hline PERCIST 30\% & $1.39(1.03-1.86)$ & 0.03 & $1.31(0.98-1.75)$ & 0.06 & $1.54(0.97-2.45)$ & 0.07 & $1.33(0.87-2.03)$ & 0.19 \\
\hline PERCIST 15\% & $1.49(1.07-2.06)$ & 0.02 & $1.97(1.40-2.78)$ & $<0.001$ & $1.71(1.0-2.92)$ & 0.05 & $1.76(1.05-2.95)$ & 0.03 \\
\hline EORTC & $1.47(1.02-2.10)$ & 0.04 & $1.62(1.12-2.34)$ & 0.01 & $1.73(0.96-3.12)$ & 0.07 & $1.56(0.91-2.68)$ & 0.11 \\
\hline CONSIST & $1.60(1.18-2.16)$ & 0.002 & $1.86(1.37-2.52)$ & $<0.001$ & $2.37(1.42-4.0)$ & 0.001 & $2.16(1.33-3.51)$ & 0.002 \\
\hline
\end{tabular}


TABLE 2. Multivariable Analyses of Clinical (age, gender, ECOG PS, KRAS, BMI) and PET-based Variables (Baseline WB-MATV and Early Metabolic Response According to Different Methods) for OS and PFS in Development and Validation sets.

\begin{tabular}{|c|c|c|c|c|c|c|c|c|}
\hline \multirow{4}{*}{ Variables } & \multicolumn{4}{|c|}{ Development Set } & \multicolumn{4}{|c|}{ Validation Set } \\
\hline & \multicolumn{2}{|l|}{ OS } & \multicolumn{2}{|l|}{ PFS } & \multicolumn{2}{|l|}{ OS } & \multicolumn{2}{|l|}{ PFS } \\
\hline & \multicolumn{4}{|c|}{ Multivariable analysis } & \multicolumn{4}{|c|}{ Multivariable analysis } \\
\hline & HR (95\% CI) & $P$ & HR (95\% CI) & $P$ & HR (95\% CI) & $P$ & HR $(95 \% \mathrm{CI})$ & $P$ \\
\hline $\begin{array}{c}\text { Baseline WB-MATV } \\
\text { (adjusted for clinical factors) }\end{array}$ & $2.56(1.90-3.44)$ & $<0.001$ & $2.00(1.51-2.66)$ & $<0.001$ & $1.87(1.17-2.97)$ & 0.005 & $1.94(1.27-2.97)$ & 0.002 \\
\hline ECOG PS & $1.47(1.12-1.94)$ & 0.006 & & & $2.01(1.08-3.74)$ & 0.03 & & \\
\hline BMI & $1.62(1.22-2.16)$ & 0.001 & & & & & & \\
\hline \multicolumn{9}{|l|}{$\begin{array}{l}\text { Early Metabolic Response } \\
\text { (adjusted for clinical factors) }\end{array}$} \\
\hline PERCIST-30\% & $1.48(1.09-2.02)$ & 0.01 & - & - & - & - & - & - \\
\hline PERCIST-15\% & $1.60(1.17-2.18)$ & 0.003 & $1.84(1.35-2.51)$ & $<0.001$ & $1.50(0.90-2.50)$ & 0.12 & $1.68(1.02-2.79)$ & 0.04 \\
\hline EORTC & $1.52(1.08-2.13)$ & 0.02 & $1.52(1.09-2.11)$ & 0.01 & $1.43(0.83-2.47)$ & 0.20 & $1.49(0.88-2.50)$ & 0.14 \\
\hline CONSIST & $1.70(1.26-2.29)$ & $<0.001$ & $1.71(1.27-2.28)$ & $<0.001$ & $1.99(1.22-3.26)$ & 0.006 & $1.98(1.24-3.15)$ & 0.004 \\
\hline ECOG PS & $1.50(1.11-2.01)$ & 0.008 & & & & & & \\
\hline BMI & $1.89(1.38-2.58)$ & $<0.001$ & & & & & & \\
\hline
\end{tabular}




\begin{tabular}{|c|c|c|c|c|c|c|c|c|}
\hline $\begin{array}{l}\text { Early Metabolic Response } \\
\text { (adjusted for clinical factors } \\
\text { and baseline WB-MATV) }\end{array}$ & & & & & & & & \\
\hline PERCIST-30\% & $1.36(1.00-1.85)$ & 0.05 & - & - & - & - & - & - \\
\hline PERCIST-15\% & $1.56(1.14-2.12)$ & 0.005 & $1.91(1.39-2.61)$ & $<0.001$ & $1.41(0.84-2.38)$ & 0.19 & $1.49(0.89-2.48)$ & 0.13 \\
\hline EORTC & $1.45(1.03-2.03)$ & 0.03 & $1.54(1.10-2.15)$ & 0.01 & $1.37(0.79-2.37)$ & 0.26 & $1.33(0.79-2.24)$ & 0.29 \\
\hline CONSIST & $1.55(1.15-2.11)$ & 0.005 & $1.64(1.23-2.20)$ & $<0.001$ & $1.79(1.08-2.95)$ & 0.02 & $1.69(1.04-2.73)$ & 0.03 \\
\hline ECOG PS & $1.38(1.02-1.86)$ & 0.035 & & & & & & \\
\hline BMI & $1.71(1.25-2.34)$ & 0.001 & & & & & & \\
\hline Baseline WB-MATV & $2.22(1.61-3.06)$ & $<0.001$ & $1.69(1.24-2.30)$ & 0.001 & $1.82(1.12-2.97)$ & 0.016 & $1.79(1.14-2.80)$ & 0.01 \\
\hline
\end{tabular}


Graphical Abstract:

Combination of metabolically active tumor volume

(WB-MATV) and early metabolic response ( $\mathrm{mR}$ )

in metastatic colorectal cancer

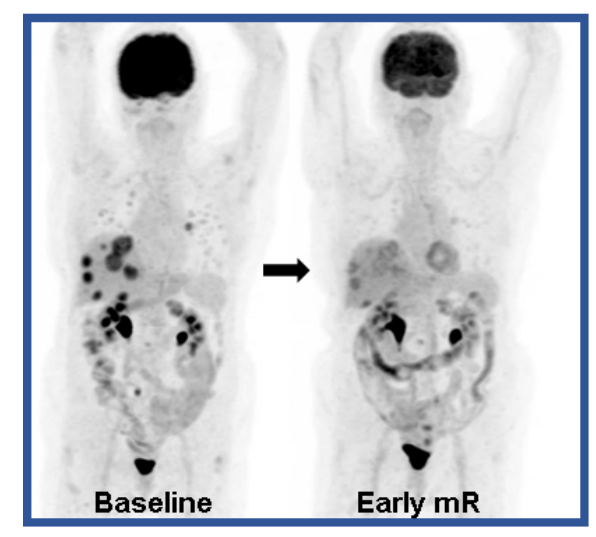

Low WB-MATV and Responder

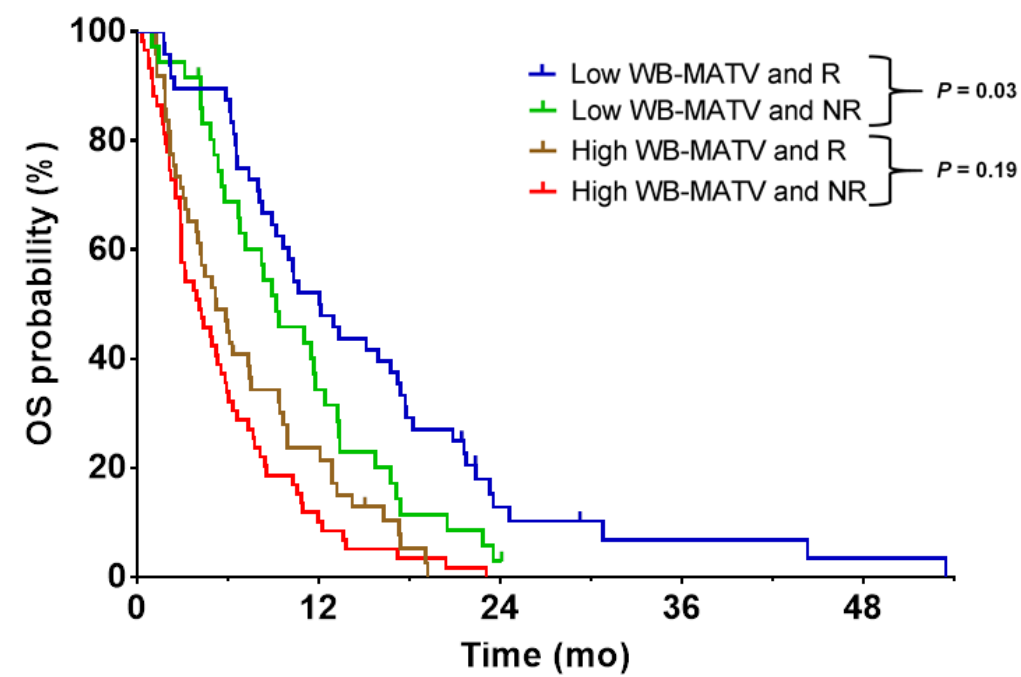

Implications:

Combining these two PET biomarkers should be implemented in future clinical trials and in clinical routine for monitoring mCRC patients under first or last-line of treatment.

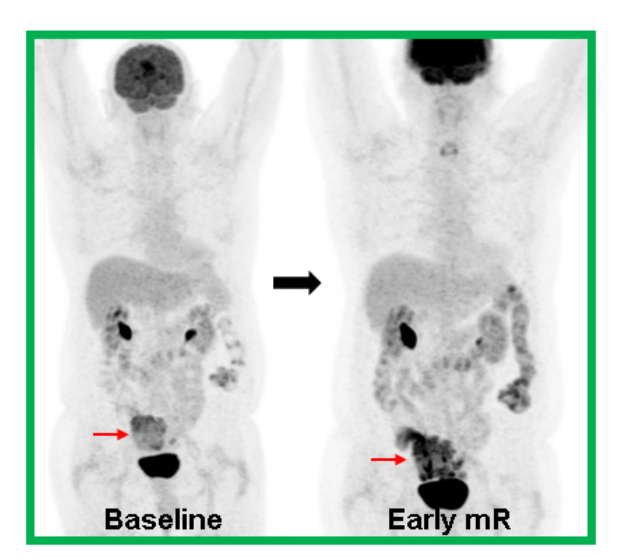

Low WB-MATV and Non-Responder 
Supplemental TABLE 1. Metabolic Response Assessment Criteria According to EORTC, PERCIST 30\%, PERCIST 15\%, and CONSIST Methodologies.

\begin{tabular}{|c|c|c|c|c|c|c|}
\hline Classification & $\begin{array}{l}\text { Definition of } \\
\text { target lesion }\end{array}$ & $\begin{array}{l}\text { Number of } \\
\text { target lesions }\end{array}$ & \multicolumn{4}{|c|}{ Response Categories } \\
\hline EORTC & \multirow{4}{*}{$\begin{array}{l}\text { SUL }_{\text {peak }} \text { of } \\
\text { baseline } \\
\text { lesions }>1.5 \mathrm{x} \\
\mathrm{SUL}_{\text {mean }}+2 \times \\
\mathrm{SD} \text {, measured } \\
\text { in a } 3 \mathrm{~cm}- \\
\text { diameter } \\
\text { sphere located } \\
\text { in healthy liver } \\
\text { tissue. If liver } \\
\text { is abnormal, } \\
\text { primary tumor } \\
\text { should have } \\
\text { uptake }>2 \times \\
\mathrm{SUL} \text { mean of } \\
\text { blood pool }+2 \\
\mathrm{x} \text { SD, } \\
\text { measured in a } \\
\text { sphere fitting } \\
\text { inside the } \\
\text { descending } \\
\text { thoracic aorta. }\end{array}$} & $\begin{array}{l}\text { Maximum } 5 \\
\text { (same lesions } \\
\text { selected on the } \\
\text { baseline scan } \\
\text { are evaluated } \\
\text { on the follow- } \\
\text { up scan) }\end{array}$ & \multirow{3}{*}{$\begin{array}{l}\text { CMR: complete } \\
\text { resolution of }{ }^{18} \mathrm{~F}- \\
\text { FDG uptake } \\
\text { within all lesions, } \\
\text { making them } \\
\text { indistinguishable } \\
\text { from the } \\
\text { surrounding tissue }\end{array}$} & $\begin{array}{l}\text { PMR: reduction of at least } \\
15 \% \text { in the sum of SUVbsa }\end{array}$ & \multirow{3}{*}{$\begin{array}{l}\text { SMD: not } \\
\text { CMR, } \\
\text { PMR, or } \\
\text { PMD }\end{array}$} & $\begin{array}{l}\text { PMD: increase of at least } 25 \% \text { in the sum of SUVbsa, } \\
\text { Or at least one lesion (even non-target) with SUVbsa increase of more than } 25 \% \text {, } \\
\text { Or appearance of at least one unequivocal new target lesion. }\end{array}$ \\
\hline PERCIST 30\% & & $\begin{array}{l}\text { The lesion with } \\
\text { the highest }{ }^{18} \mathrm{~F} \text { - } \\
\text { FDG uptake } \\
\text { identified on }\end{array}$ & & $\begin{array}{l}\text { PMR: reduction of at least } 30 \\
\% \text { in SULpeak and an absolute } \\
\text { drop of } 0.8 \text { SULpeak units in } \\
\text { the hottest lesion }\end{array}$ & & \multirow{2}{*}{$\begin{array}{l}\text { PMD: increase of at least } 30 \% \text { in SULpeak and an absolute increase of } 0.8 \text { SULpeak } \\
\text { units, } \\
\text { Or: } 75 \% \text { increase in TLG in a single lesion with no decrease in SULpeak (only if } \\
\text { the baseline MATV of this lesion is }>4 \mathrm{cc} \text { ) } \\
\text { Or: appearance of at least one unequivocal new target lesion. }\end{array}$} \\
\hline PERCIST 15\% & & $\begin{array}{l}\text { the baseline } \\
\text { and follow-up } \\
\text { scans (not } \\
\text { always the } \\
\text { same lesion) }\end{array}$ & & $\begin{array}{l}\text { PMR: reduction of at least } 15 \\
\% \text { in SUL peak and an absolute } \\
\text { drop of } 0.4 \text { SUL } \\
\text { the hottest lesion }\end{array}$ & & \\
\hline CONSIST & & $\begin{array}{l}\text { Unlimited } \\
\text { (same lesions } \\
\text { selected on the } \\
\text { baseline scan } \\
\text { are evaluated } \\
\text { on the follow- } \\
\text { up scan) }\end{array}$ & $\begin{array}{l}\text { Responder: all } \\
\text { lesions } \\
\text { responding with a } \\
\text { decrease of } \\
\text { SUL }_{\max } \geq 15 \%\end{array}$ & \multicolumn{3}{|c|}{$\begin{array}{l}\text { Non-responder: at least one non-responding lesion }\left(\mathrm{SUL}_{\max } \text { decrease }<15 \%\right) \text {, } \\
\left.\text { Or at least one progressive lesion (SUL } \mathrm{SLx}_{\max } \text { increase }>15 \%\right) \text {, } \\
\text { Or at least one unequivocal new target lesion. }\end{array}$} \\
\hline
\end{tabular}


Supplemental TABLE 2. Patient and Disease Characteristics of the Evaluable Population.

\begin{tabular}{|r|c|cc|}
\hline & $\begin{array}{c}\text { Belgian cohort } \\
(\boldsymbol{N}=\mathbf{2 2 4})\end{array}$ & $\begin{array}{c}\text { Italian external } \\
\text { validation } \\
\text { cohort }\end{array}$ \\
(N= 109)
\end{tabular}




\begin{tabular}{|c|c|c|c|c|}
\hline \multirow[b]{2}{*}{ Left-sided colon } & \multicolumn{2}{|c|}{$\begin{array}{l}\text { Belgian cohort } \\
\quad(N=224)\end{array}$} & \multicolumn{2}{|c|}{$\begin{array}{c}\text { Italian external } \\
\text { validation } \\
\text { cohort } \\
(N=109)\end{array}$} \\
\hline & 81 & $36 \%$ & 29 & $27 \%$ \\
\hline Rectum & 65 & $29 \%$ & 19 & $17 \%$ \\
\hline Missing data & 31 & $14 \%$ & 33 & $30 \%$ \\
\hline No & 26 & $18 \%$ & 40 & $37 \%$ \\
\hline Yes & 115 & $82 \%$ & 67 & $61 \%$ \\
\hline Missing data & & & 2 & $2 \%$ \\
\hline $\begin{array}{l}\text { Primary tumor } \\
\text { radiotherapy }(n) \text { * }\end{array}$ & & & & \\
\hline No & 115 & $82 \%$ & 98 & $90 \%$ \\
\hline Yes & 26 & $18 \%$ & 7 & $6 \%$ \\
\hline Missing data & & & 4 & $4 \%$ \\
\hline $\begin{array}{l}\text { Baseline } \\
\text { WB-MATV }\left(\mathrm{cm}^{3}\right)\end{array}$ & & & & \\
\hline Median (min-max) & 160( & 448) & $149(3$ & 3773) \\
\hline$<100$ & 90 & $40 \%$ & 47 & $43 \%$ \\
\hline$\geq 100$ & 134 & $60 \%$ & 62 & $57 \%$ \\
\hline $\begin{array}{l}\text { Early metabolic } \\
\text { response assessment } \\
(n)\end{array}$ & & & & \\
\hline NR & 95 & $49 \%$ & 35 & $37 \%$ \\
\hline $\mathrm{R}$ & 97 & $51 \%$ & 59 & $63 \%$ \\
\hline
\end{tabular}




\begin{tabular}{|l|c|c|}
\hline & $\begin{array}{c}\text { Belgian cohort } \\
(\boldsymbol{N}=\mathbf{2 2 4})\end{array}$ & $\begin{array}{c}\text { Italian external } \\
\text { validation } \\
\text { cohort } \\
(\boldsymbol{N}=\mathbf{1 0 9})\end{array}$ \\
\hline $\begin{array}{l}\text { Median OS since } \\
\text { treatment start } \\
\text { (95\% CI) (months) }\end{array}$ & $6.7(5.7$ to 7.7$)$ & $\begin{array}{c}25.2(20.9 \text { to } \\
27.2)\end{array}$ \\
$\begin{array}{l}\text { N Deaths } \\
\text { Median PFS since } \\
\text { treatment start } \\
\text { (95\% CI) (months) }\end{array}$ & $2.9(2.4$ to 3.3) & $9.7(8.4$ to 11.5) \\
N PFS events & 224 & 109 \\
\hline
\end{tabular}

* Data available only from the RegARd-C cohort concerning the Belgian cohort. 
Supplemental TABLE 3. Diagnostic Performance in Terms of Harrell's c-index, for OS and PFS in Development and Validation Sets, Considering Clinical (age, gender, ECOG PS, KRAS, BMI $\geq 25$ ) and PET-based Variables (Baseline WB-MATV and Metabolic Response).

\begin{tabular}{|c|c|c|c|c|c|c|c|c|}
\hline \multirow{3}{*}{ Model } & \multicolumn{4}{|c|}{ Development Set } & \multicolumn{4}{|c|}{ Validation Set } \\
\hline & \multicolumn{2}{|c|}{ OS } & \multicolumn{2}{|c|}{ PFS } & \multicolumn{2}{|c|}{ OS } & \multicolumn{2}{|c|}{ PFS } \\
\hline & c-index & $S E$ & c-index & $S E$ & c-index & $S E$ & c-index & $S E$ \\
\hline Clinical factors and baseline WB-MATV & 0.693 & 0.021 & 0.613 & 0.023 & 0.618 & 0.035 & 0.638 & 0.031 \\
\hline \multicolumn{9}{|l|}{ Clinical factors and metabolic response } \\
\hline Clinical factors and PERCIST 30\% & 0.635 & 0.022 & 0.571 & 0.025 & 0.598 & 0.034 & 0.613 & 0.029 \\
\hline Clinical factors and PERCIST $15 \%$ & 0.638 & 0.021 & 0.601 & 0.023 & 0.601 & 0.034 & 0.612 & 0.031 \\
\hline Clinical factors and EORTC & 0.631 & 0.021 & 0.567 & 0.024 & 0.596 & 0.033 & 0.607 & 0.030 \\
\hline Clinical factors and CONSIST & 0.646 & 0.022 & 0.609 & 0.025 & 0.626 & 0.034 & 0.642 & 0.029 \\
\hline \multicolumn{9}{|c|}{ Clinical factors and baseline $W B-M A T V$ and metabolic response } \\
\hline Clinical factors and WB-MATV and PERCIST 30\% & 0.696 & 0.021 & 0.624 & 0.023 & 0.632 & 0.034 & 0.650 & 0.031 \\
\hline Clinical factors and WB-MATV and PERCIST 15\% & 0.700 & 0.020 & 0.638 & 0.022 & 0.633 & 0.035 & 0.650 & 0.032 \\
\hline Clinical factors and WB-MATV and EORTC & 0.695 & 0.020 & 0.623 & 0.022 & 0.632 & 0.035 & 0.650 & 0.032 \\
\hline Clinical factors and WB-MATV and CONSIST & 0.700 & 0.021 & 0.641 & 0.022 & 0.650 & 0.035 & 0.667 & 0.032 \\
\hline
\end{tabular}


Supplemental FIGURE 1. Example of a patient subject to differences in response assessment following PERCIST and EORTC methodologies. PET maximum-intensity projections images of a patient at baseline (A) and early time-point (B) who was classified as responder (partial metabolic response) according to EORTC and as non-responder (stable metabolic disease) according to PERCIST. The PERCIST classification was based on the two highest SUL peak lesions (red arrows targeting a retroperitoneal lesion with a SULpeak of $6.5 \mathrm{~g} / \mathrm{mL}$ at baseline and $5.6 \mathrm{~g} / \mathrm{mL}$ at early time-point) giving a difference of $-14.3 \%$ between these two lesions. Conversely, EORTC classification was based on the difference between the sum of the $\mathrm{SUV}_{\text {bsa }}$ lesions (blue and red arrows) at baseline $\left(6.6 \mathrm{~cm}^{2} / \mathrm{mL}\right)$ and early time-point $\left(4.5 \mathrm{~cm}^{2} / \mathrm{mL}\right) \mathrm{giving}$ a difference of $32.1 \%$ between these sums.

A

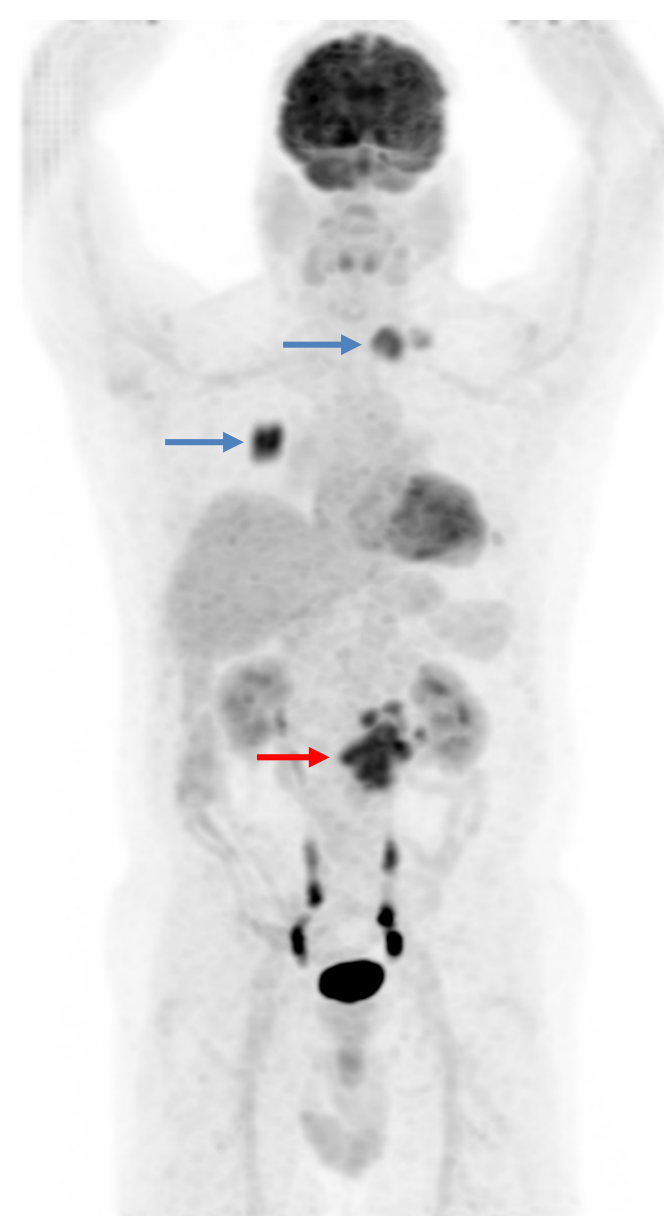

Baseline
B

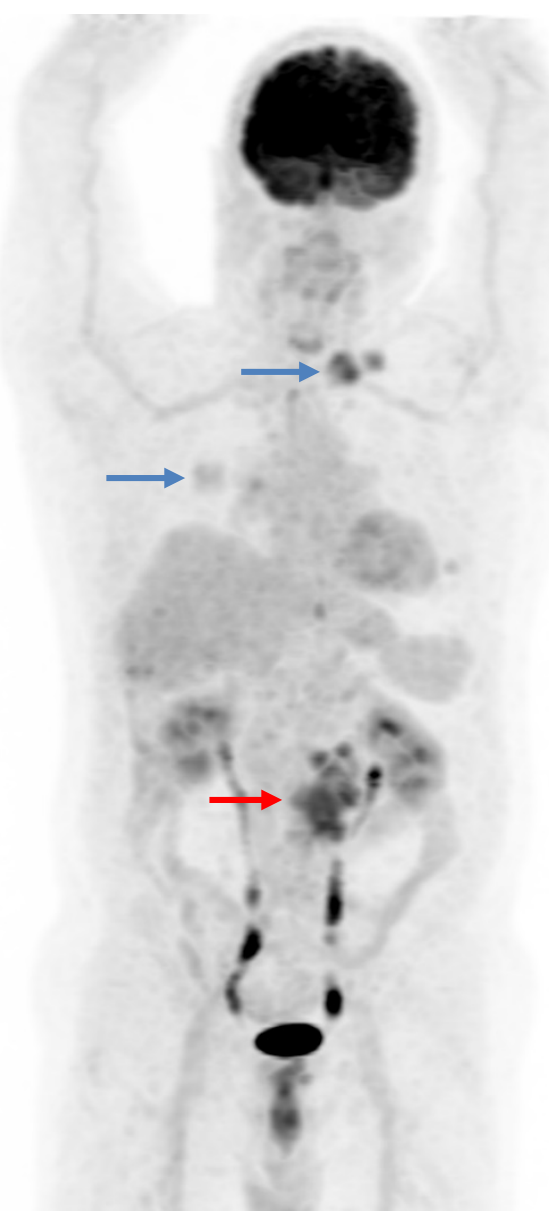

Early 